\title{
A regulatory network governing Gata1 and Gata2 gene transcription orchestrates erythroid lineage differentiation
}

\author{
Takashi Moriguchi • Masayuki Yamamoto
}

Received: 12 February 2014/Revised: 4 March 2014/Accepted: 4 March 2014/Published online: 18 March 2014

(c) The Japanese Society of Hematology 2014

\begin{abstract}
GATA transcription factor family members GATA1 and GATA2 play crucial roles in the regulation of lineage-restricted genes during erythroid differentiation. GATA1 is indispensable for survival and terminal differentiation of erythroid, megakaryocytic and eosinophilic progenitors, whereas GATA2 regulates proliferation and maintenance of hematopoietic stem and progenitor cells. Expression levels of GATA1 and GATA2 are primarily regulated at the transcriptional level through auto- and reciprocal regulatory networks formed by these GATA factors. The dynamic and strictly controlled change of expression from GATA2 to GATA1 during erythropoiesis has been referred to as GATA factor switching, which plays a crucial role in erythropoiesis. The regulatory network comprising GATA1 and GATA2 gives rise to the stage-specific changes in Gatal and Gata2 gene expression during erythroid differentiation, which ensures specific expression of early and late erythroid genes at each stage. Recent studies have also shed light on the genome-wide binding profiles of GATA1 and GATA2, and the significance of epigenetic modification of Gatal gene during erythroid differentiation. This review summarizes the current understanding of network regulation underlying stagedependent Gatal and Gata2 gene expressions and the functional contribution of these GATA factors in erythroid differentiation.
\end{abstract}

T. Moriguchi · M. Yamamoto $(\bowtie)$

Department of Medical Biochemistry, Graduate School of

Medicine, Tohoku University, 2-1 Seiryo-machi, Aoba-ku,

Sendai 980-8575, Japan

e-mail: masiyamamoto@med.tohoku.ac.jp
Keywords GATA1 - GATA2 - Transgenic mouse · Differentiation · Erythroid · BAC (bacterial artificial chromosome)

\section{Introduction}

The mechanisms underlying lineage-specific hematopoietic differentiation have been studied extensively to elucidate how particular transcription factor networks influence each differentiation process. Upon hematopoietic differentiation, lineage-specific gene expression programs are directed through such transcription factor networks to generate the diversity of cellular function in each hematopoietic lineage.

The GATA family of transcription factors, which comprises six members (GATA1 through GATA6) in mammals, is one of the key regulators orchestrating such transcription factor networks [1-3]. GATA proteins bind most avidly to the consensus motif (T/A)GATA(A/G) through two characteristic zinc-finger motifs, which are conserved among the six GATA family members $[4,5]$. Of these, GATA1-3 constitute the "hematopoietic GATA" subfamily due to their prominent expression in hematopoietic cells [5-7]. GATA1 is a prototypical transcription factor that promotes hematopoietic differentiation in erythroid, eosinophilic and megakaryocytic lineages. A series of gene-targeting studies in mice revealed that GATA1 is essential for the differentiation of erythroid cells [8-10]. In contrast, GATA2, which is predominantly expressed in hematopoietic stem and progenitor cells, regulates their proliferation and maintenance $[11,12]$.

Regulatory interactions between these two GATA factors during erythropoiesis have long been examined exploiting a variety of experimental approaches. A growing body of data has provided substantial insight into the 
regulatory functions of each cis-element in the Gatal and Gata2 genes. In the present review, we summarize recent topics that address the molecular mechanisms of the regulatory network underlying lineage-specific Gatal and Gata2 gene expression and the intimate cooperation of these transcription factors during erythropoiesis.

\section{Gata1 gene structure and Gata1 hematopoietic regulatory domain}

The murine Gatal gene contains two non-coding first exons, termed IT and IE, and five coding exons (Fig. 1a) $[6,13,14]$. The proximal IE exon directs Gatal expression in hematopoietic cells $[13,15]$, while the distal IT exon primarily directs Gatal expression in testicular Sertoli cells $[16,17]$. The regulation of Gatal gene was initially studied using erythroid cell lines, MEL and K562 [18, 19], and was subsequently studied in greater detail through in vivo transgenic reporter mouse approaches $[14,20]$. We found that an $8.5-\mathrm{kb}$ Gatal genomic region covering $3.9-\mathrm{kb}$ upstream of exon IE to the second exon harbors sufficient regulatory information to induce hematopoietic lineagespecific expression of $\beta$-galactosidase reporter in both yolk sac-derived primitive and fetal liver-derived definitive hematopoietic cells in transgenic mouse assays [14, 20]. This region is referred to as the Gatal hematopoietic regulatory domain (G1HRD; Fig. 1b) and utilized as an extremely useful genetic tool that directs erythroid- and megakaryocyte-specific expression of various transgenes [21-23].

We have generated a mutant mouse line bearing a Gatal knockdown allele, Gatal.05, in which Gatal mRNA

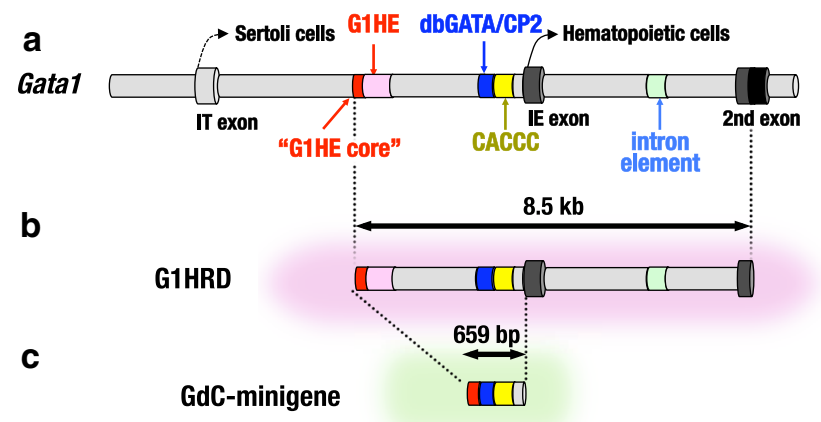

Fig. 1 a Mouse Gatal gene harbors two first exons (IT, testis exon 1; IE, hematopoietic exon 1) and four regulatory modules (G1HE core, dbGATA/CP2, CACCC and first intron element). b G1HRD (Gata1 Hematopoietic Regulatory Domain) carries a $3.9-\mathrm{kb} 5^{\prime}$-flanking sequence, IE exon, a 4.2-kb first intron, and second exon non-coding sequences. c A 659-bp GdC minigene containing G1HE, double GATA and CACCC motifs in the G1HRD. Note that this minigene elicits sufficient regulatory activity to direct erythrocyte-specific Gata1 expression upon equipping the first intron element expression is suppressed to approximately $5 \%$ of the wildtype level [9]. Hemizygous (Gatal ${ }^{\mathrm{G} 1.05 / \mathrm{Y}}$ ) male embryos harboring Gatal.05 knockdown allele succumb around embryonic day 10.5 (E10.5) due to severe anemia, indicating that $5 \%$ of GATA1 production is insufficient to support embryonic erythropoiesis [9]. Importantly, when GATA1 is expressed under the direction of G1HRD, transgenic GATA1 restores hematopoiesis in Gatal.05 knockdown mice, thereby rescuing knockdown mice from embryonic lethality [10], which indicates that G1HRD harbors sufficient regulatory information to support Gatal gene expression for physiological hematopoiesis.

\section{Sequences and mechanisms that regulate Gata1 transcription}

The G1HRD-based transgenic rescue system has been extensively used for the dissection of functional domains of GATA1 protein and the functional evaluation of hematopoietic disease-related mutants of GATA1 [24, 25]. A number of studies have addressed the regulatory mechanisms by which Gatal gene expression is regulated through the use of a G1HRD-based $\beta$-galactosidase reporter transgenic mouse system. These analyses have revealed the existence of multiple cis-regulatory elements that confer lineage- and stage-specific Gatal gene expression [14, 20, 26, 27].

Of the cis-acting regulatory elements, Gatal hematopoietic enhancer (G1HE), which is located at the $5^{\prime}$ end of G1HRD (Fig. 1a), is one such regulatory region. In transgenic reporter mouse assays, a deletion of $1.3-\mathrm{kb}$ region from the $5^{\prime}$ end of G1HRD (including G1HE) completely abolished $L a c Z$ reporter gene expression in erythroid and megakaryocytic lineage cells in the fetal liver hematopoietic cells $[14,20]$. Further dissection of G1HE revealed that a 235-bp region at the most $5^{\prime}$ end of G1HE, which is designated as the G1HE core region, is essential for the activity of G1HE element (Fig. 1a) [20, 26]. The G1HE core region contains an evolutionarily conserved GATAbinding motif. A substitution mutation of the GATA motif in the context of G1HRD-based vector significantly decreased the expression of LacZ reporter in fetal liver hematopoietic cells, underscoring the importance of the GATA site for hematopoietic GATA1 expression [20].

Also of interest, two adjacent CACCC boxes are located at around -201 to $-186 \mathrm{bp}$ in close proximity to the IE promoter (Fig. 1a). This region was originally reported as a DNase I hypersensitive (HS) site [18]. While the contribution of these CACCC boxes to the Gatal gene expression was shown by transient reporter transfection assays in erythroid cell lines in the initial stage of Gatal study [18], the physiological significance of these elements in vivo 
remains unclear. One transgenic reporter mouse experiment has shown that mutation in one of the two CACCC boxes hardly affects the G1HRD-directed LacZ reporter expression, suggesting that one of the two CACCC elements may suffice for hematopoietic Gatal expression [26, 27]. The most plausible trans-activating factor that binds to these elements would appear to be Erythroid Krüppel-like Factor (EKLF or KLF1) [28]; however, no direct evidence for this assertion has been reported.

Thirdly, a palindromic GATA-binding site (dbGATA) is located 700-bp $5^{\prime}$ to the IE exon (Fig. 1a), which has eightfold higher binding affinity than a single GATA site $[29,30]$. Targeted deletion of the dbGATA site $(\triangle$ dbGATA) leads to selective loss of the eosinophil lineage [31], while the significance of the dbGATA site for Gatal gene expression in erythroid and megakaryocytic progenitor cells has largely been unexplored. In this regard, recent studies using the G1HRD-based transgenic reporter mouse assay have revealed that the dbGATA site is essential for GATA1 expression in fetal liver hematopoietic cells [26, 27].

CP2 is a ubiquitously expressed transcription factor belonging to the Drosophila grainyhead-like gene family [32]. CP2 participates in the regulation of $\alpha$ - and $\beta$-globin gene expression [32, 33]. CP2-binding sites are located in close proximity to GATA-binding sites in the promoter and enhancer region of erythroid genes (e.g., GATA1, EKLF, and p45 NF-E2). Indeed, an evolutionally conserved CP2binding site lies adjacent to the dbGATA site in the Gatal regulatory region (Fig. 1a) [34]. In the G1HRD-directed reporter assay system, both the dbGATA- and CP2-binding sites participate cooperatively in Gatal gene regulation in erythroid cells [27]. Direct interactions between CP2 and GATA1 may be responsible for erythroid-specific regulation of the Gatal gene [34].

Finally, an element localized in the first intron appears to be essential for definitive hematopoietic cell-specific G1HRD transgene expression in mouse fetal liver, whereas this region is dispensable for Gatal gene expression in yolk sac primitive erythroid cells [14]. In the transgenic reporter analysis, Gatal expression in definitive erythroid cells and megakaryocytes requires a 320-bp region in the Gatal first intron [26], which contains GATA motifs and AP1 repeats (Fig. 1a). Seven highly conserved GATA motifs, and two alternative transcription start sites $\left(\mathrm{IE}_{\mathrm{b}}\right.$ and $\mathrm{IE}_{\mathrm{c}}$ ), are also identified in this region [15].

\section{GdC minigene: minimal sufficient regulatory element for hematopoietic Gatal expression}

Efforts to isolate minimal cis-acting element sufficient for the hematopoietic Gatal gene expression led to the identification of a 659-bp small DNA fragment containing the aforementioned three core elements [i.e., G1HE, double GATA and CACCC (+CP2) motifs in the G1HRD]. This fragment elicits regulatory activity sufficient to direct Gatal expression in yolk sac erythroid cells [26], and is referred to as Gatal GdC minigene (Fig. 1c). The GdC-minigene fragment is indeed capable of functionally replacing the $3.7-\mathrm{kb} 5^{\prime}$ upstream regulatory region of Gatal gene by directing the hematopoietic lineage-specific gene expression in the context of a Gatal bacterial artificial chromosome (BAC)-based transgenic mouse assay [35]. These three regulatory elements are thus crucial for hematopoietic Gatal gene expression.

\section{Dynamic expression profiles of GATA1 and GATA2 during erythropoiesis}

Throughout the erythroid differentiation process, GATA1 orchestrates dramatic changes in the expression of a series of genes, which promote essential steps required for the proliferation and differentiation of erythroid progenitors. In the early stage of erythroid commitment, GATA1 expression is first initiated at the common myeloid progenitor (CMP) stage (Fig. 2). Subsequently, GATA1 expression increases and reaches a peak when erythroid-committed progenitors give rise to proerythroblasts [36, 37]. When proerythroblasts enter terminal erythroid differentiation, GATA1 directly activates a number of erythroid-affiliated genes, i.e. $\beta$-globin, Alas 2 and Gata1 itself, and represses a number of genes essential for progenitor proliferation in the early stage of hematopoiesis, including Gata2, c-Kit, c-Myb and c-Myc [7]. From the late erythroblast stage onward, GATA1 expression levels decrease toward maturation of red blood cells [36, 37].

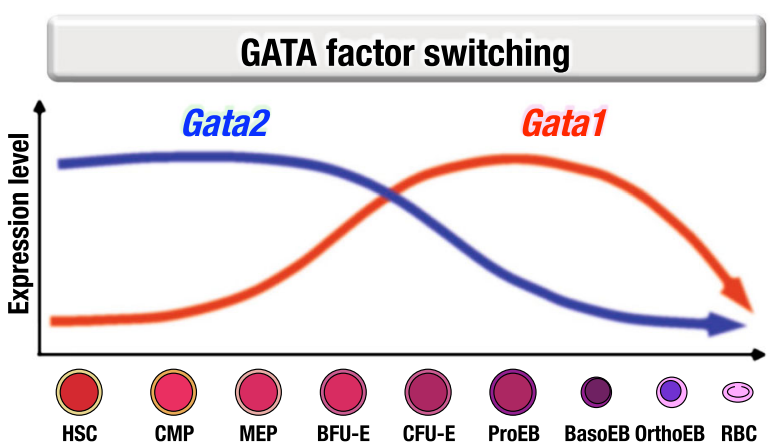

Fig. 2 Reciprocal expression profiles of GATA1 and GATA2 during erythropoiesis. GATA1 expression is initiated at common myeloid progenitors (CMP) stage and reaches a peak at the proerythroblast (ProEB) stage. From the late erythroblast stage onward, GATA1 expression levels decrease toward maturation of red blood cell (RBC). GATA2 is preferentially expressed in hematopoietic stem cell (HSC) and hematopoietic progenitor cells, including CMP, megakaryoerythroid progenitor (MEP), and burst forming unit-erythroid (BFUE). GATA2 expression is suppressed by the increase of GATA1 activity from CFU-E (colony forming unit-erythroid) stage onward 
This series of changes in GATA1 expression level is essential for erythropoiesis. Indeed, forced GATA1 expression driven by human $\beta$-globin gene promoter in the terminal erythroid differentiation stage leads to defective erythroid cell maturation, indicating that aberrant GATA1 activity inhibits terminal erythroid differentiation [38].

In contrast to GATA1, GATA2 is preferentially expressed in hematopoietic stem cells (HSC) and early hematopoietic progenitor cells (HPC; Fig. 2). GATA2 is essential for the development and maintenance of these fractions [11, 39-41]. GATA2 haploinsufficiency impairs the quality of both embryonic and adult HSC, and eventually leads to a reduction of early HSC population [12]. Two independent studies demonstrated that Gata2 4th intron regulatory elements (Gata2 VE; vascular enhancer/+9.5-kb GATA motifs) [42] are crucial for GATA2 expression in HSCs [43, 44]. Of note, a heterozygous 28-bp deletion within this element was identified in a patient with MonoMAC syndrome, an autosomal dominant condition that features recurrent mycobacterial infection associated with deficiencies of monocytes, B cells and NK cells, and myelodysplasia [43]. Gata2 expression from the mutated allele was reduced in the peripheral blood monocyte of the patient, suggesting a causal contribution of the $+9.5-\mathrm{kb}$ deletion to the diminished GATA2 level and the subsequent pathogenesis of MonoMAC syndrome.

\section{GATA switching underlies erythropoiesis}

Hematopoietic stem and progenitor cell compartments abundantly express GATA2. The Gata2 gene is trans-activated by the binding of GATA2 itself to multiple auto-regulatory GATA sites at $-77 \mathrm{~kb},-3.9 \mathrm{~kb},-2.8 \mathrm{~kb},-1.8 \mathrm{~kb}$, and the 4th intronic Gata2 VE/+9.5-kb regions relative to the Gata2 IS exon (Fig. 3a, b). GATA2 also participates in the activation of Gatal gene expression at the initial phase of erythroid/megakaryocytic differentiation (Fig. 3a). During terminal differentiation of erythroblasts, Gatal gene expression is maximized by GATA1-mediated auto-regulatory loop through the GATA sites in the Gatal gene hematopoietic regulatory region. Thereafter, the increased level of GATA1 displaces GATA2 at the multiple GATAbinding sites of the Gata 2 gene and suppresses its expression [3, 45, 46]. This biphasic transition of dominant GATA factors has been referred to as the "GATA Factor Switching", which is essential for proper erythropoiesis.

As described earlier, G1HRD-directed transgenic GATA1 expression completely restores hematopoiesis in the Gatal.05 knockdown mice; the rescued mice are referred to as G1R mice [10]. G1R mice survive to adulthood without any hematological abnormality throughout their lives. Given this result, another transgenic rescue

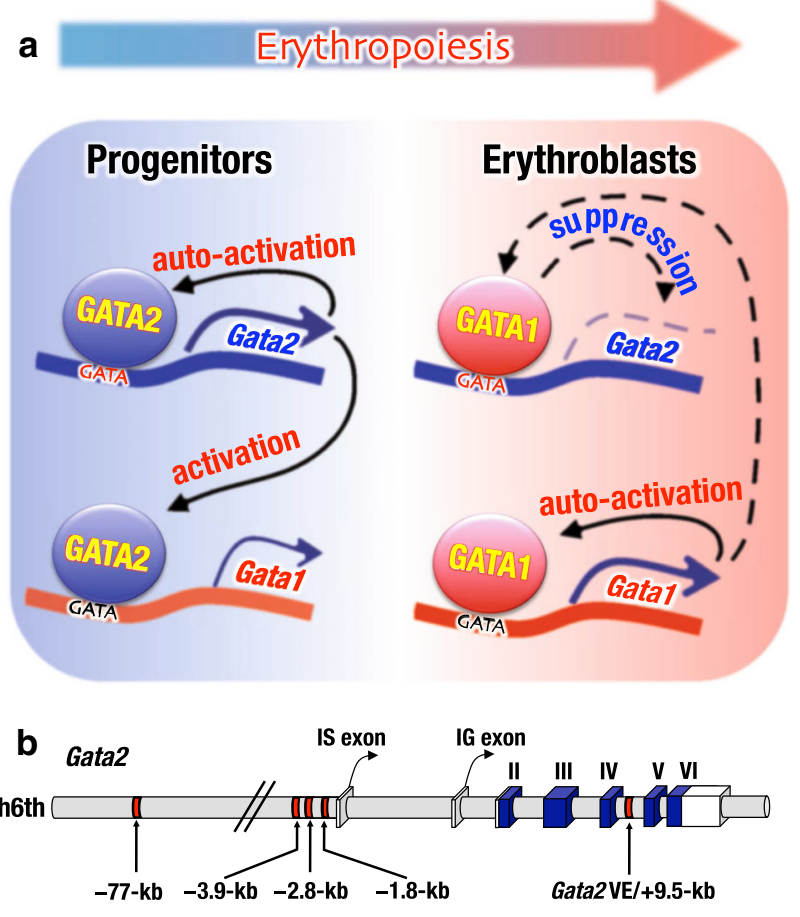

Fig. 3 a GATA factor switching. GATA2 auto-activates Gata2 expression and transactivates Gatal expression in hematopoietic progenitors (left). In erythroblast differentiation, GATA1 autoactivates Gatal expression (right) and Gata2 expression is decreased due to GATA1-mediated suppression. b Gata2 gene harbors two distinct first exons. The $5^{\prime}$-distal first exon (IS) is specifically expressed in hematopoietic and neural cells, whereas the geneproximal first exon (IG) is transcribed in almost all Gata2-expressing cells [40, 41]. Blue boxes depict coding exons. GATA-switching site at $-77,-3.9,-2.8$, and $-1.8 \mathrm{~kb}$ and the 4 th intronic $+9.5 \mathrm{~kb}$ (Gata2 $\mathrm{VE}$; vascular enhancer) regions are indicated

assays have been conducted by crossing G1HRD-GATA2 transgenic mice to Gatal.05 knockdown mice to address physiological importance of GATA1/GATA2 balance in vivo. G1HRD-directed GATA2 is found to sustain embryonic hematopoiesis and rescue Gatal.05 mice (G2R mice) from embryonic lethality. However, G2R mice exhibit significant anemia in later adult stages [10]. In the G2R mouse, GATA2 expression recapitulates that of GATA1 and substitutes for GATA1 function in the Gata1.05 knockdown mice. It thus appears that normal GATA factor switching from GATA2 to GATA1 does not occur in G2R mice. These results thus indicate that the GATA factor switching is essential to fully develop hematopoietic system in adult stage.

\section{Binding profiles of GATA1 and GATA2 to genome-wide GATA motifs in a proerythroblast cell line}

A proerythroblast-like cell line GAK14 (Gatal gene knockdown c-Kit ${ }^{+}$leukemia-derived cell line 14) has been 
established from erythroleukemia cells spontaneously developed in Gatal gene knockdown heterozygous female $\left(\right.$ Gatal $\left.^{\mathrm{G} 1.05 /+}\right)$ mice [47]. GAK14 cells actively proliferate as immature erythroid progenitors. GAK14 cells abundantly express GATA2, while GATA1 expression is significantly diminished in these cells. When exogenous GATA1 activity is introduced into GAK14 cells, erythroid differentiation of the cells is resumed, but GATA2 expression is concomitantly suppressed. The rescued cells are referred to as GKG cells. GAK14 and GKG cells serve as cellular models for two different stages of erythroid progenitors.

Taking advantage of an in vitro erythroid differentiation system, GATA2 and GATA1 ChIP-chip analyses have been conducted to examine binding profiles of GATA1 and GATA2 to genome-wide cis-regulatory GATA motifs during erythropoiesis [48]. Through this analysis, GATAbinding motifs were classified into three categories based on binding profiles of GATA1 and GATA2 (Fig. 4). In this study, GATA sites that are exclusively occupied by GATA2 in GAK14 cells were categorized as GATA2preferred sites (top panel), and GATA sites showing both GATA2 binding in GAK14 cells and GATA1 occupancy in GKG cells were designated as GATA-switching sites (middle panel). GATA sites characterized by exclusive GATA1 occupancy in GKG cells, but lacking GATA2 binding in GAK14 cells, were classified as GATA1-preferred sites (bottom panel).

Intriguingly, GATA2-preferred sites typically contain GATA-binding sites in progenitor marker genes, such as CD34. Gata2, Gatal, Zfpml coding for FOG1, and Ppox were identified as GATA-switching site genes, suggesting that GATA1 either activates or represses these target genes through such GATA-sites. GATA1-preferred sites include terminal erythroid-affiliated genes, including $\mathrm{Hbb}, \mathrm{Nfe2}$, and $\mathrm{Klfl}$.

This ChIP-chip analysis demonstrates that the preoccupancy by GATA2 at the GATA-switching sites promotes subsequent smooth GATA1 binding at the same site in comparison with the GATA1-preferred sites [48]. Initial GATA2 binding may change the chromatin configuration around the GATA-switching site, so that GATA1 can easily access GATA-switching sites. Taking advantage of this quick transition, GATA factor switching may facilitate immediate changes in gene expression profiles during erythroid commitment. The mechanism by which each GATA factor binds to a specific set of GATA motifs remains unexplored.

Another genome-wide ChIP-seq analysis using K562 human leukemia cells has been reported. Consistent with our ChIP-chip analysis, the ChIP-seq analysis has revealed that $65 \%$ of GATA1-binding sites are co-occupied by both GATA1 and GATA2 [49]. While K562 cells express both GATA1 and GATA2, the key regulatory step seems to be

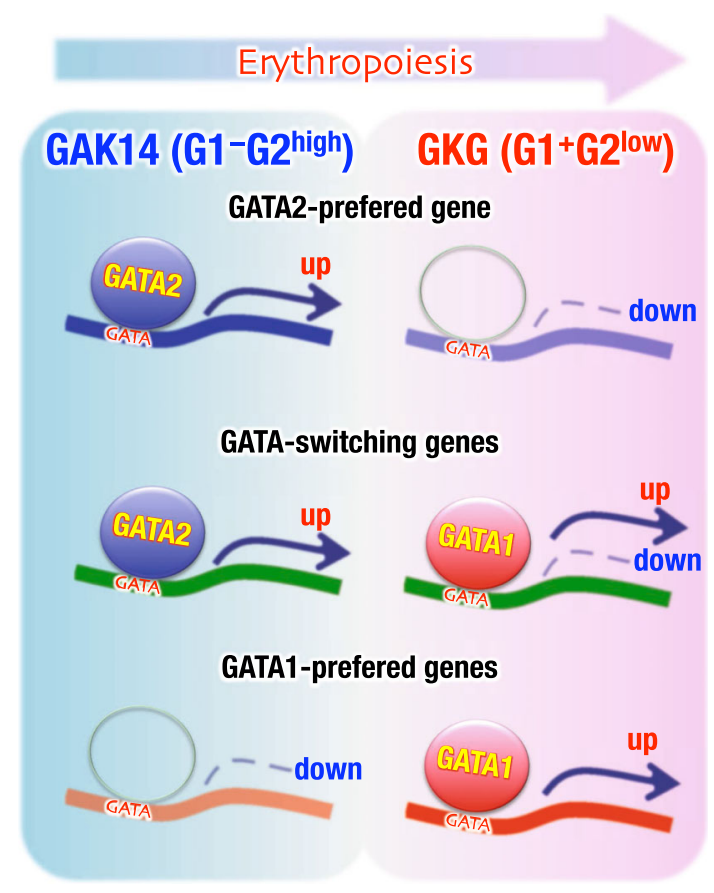

Fig. 4 Three binding profiles of GATA1 and GATA2 to hematopoietic GATA motifs. A proerythroblast-like cell line GAK14 has been established from erythroleukemia cells developed in Gatal knockdown mice. GAK14 cells abundantly express GATA2. When exogenous GATA1 is introduced into GAK14 cells, erythroid differentiation of the cells is resumed and the rescued cells are referred to as GKG cells. GAK14 and GKG cells are taken to represent two different stages of erythroid progenitors. Using these two cell lines, three binding profiles of GATA1 and GATA2 are identified; top GATA2-preferred sites are exclusively occupied by GATA2 in GAK14 cells. Middle GATA-switching sites show GATA2 binding in GAK14 and GATA1 binding in GKG cells. GATA1 confers either up-regulation or down-regulation of target genes through the GATA-switching sites. Bottom GATA1-preferred sites are characterized with GATA1 occupancy in GKG cells, but lacking GATA2 peaks in GAK14 cells

the switch from GATA2 to GATA1 during erythroid differentiation, suggesting that GATA switching may occur at the vast majority of the GATA1/GATA2 co-occupied sites identified in K562 cells.

\section{Gata1 BAC system for gene transcription study}

Although the G1HRD transgenic mouse system provides insights into Gatal gene regulation, there are several limitations to this approach. Firstly, G1HRD activity is often subjected to position-effect variegation $[14,36]$. Secondly, G1HRD appears to be functionally insufficient to fully recapitulate Gatal gene expression profile, especially insufficient to direct the reporter gene expression in c-Kitpositive early hematopoietic progenitors [36]. To circumvent these limitations, we developed a BAC (RP23- 


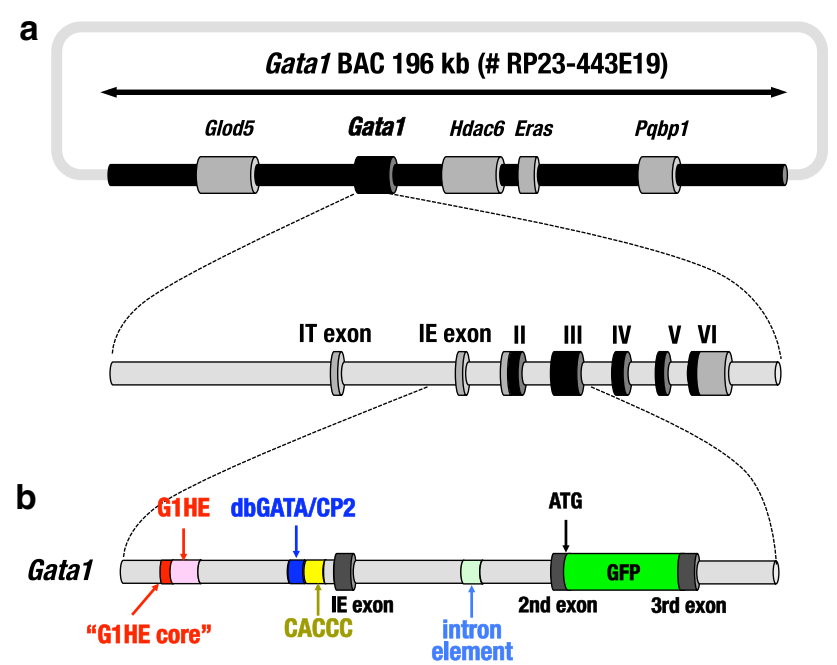

Fig. 5 a Gatal bacterial artificial chromosome (BAC). Clone \#RP23-443E19 harbors a 196-kb genomic fragment including the Gatal gene and flanking sequences. b GFP reporter gene is inserted between 2 nd and 3rd exons in-frame with the translation initiation site by means of homologous recombination in E. coli strain EL250. Four regulatory modules (G1HE core, dbGATA/CP2, CACCC and first intron element) are indicated

443E19) carrying an approximately 196-kb Gatal genomic fragment including the Gatal gene and flanking sequences (Fig. 5a). The transgenic GFP reporter expression directed by the regulatory influences of the Gatal BAC (G1BAC) DNA sequences recapitulates more faithfully the endogenous Gatal gene expression profile than does the G1HRDbased transgene (Fig. 5b) [37, 50]. Indeed, the G1BAC directs GFP fluorescence highly in the c-Kit ${ }^{+}$progenitors in which endogenous GATA1 is expressed. Moreover, the G1BAC-directed transgenic mouse system confers integration site-independent and copy number-dependent gene expression $[37,50]$. Thus, the BAC clone evidently contains a more comprehensive set of Gatal gene regulatory elements that mediate proper spatiotemporal expression of Gatal.

The elaborate G1BAC-based transgenic reporter assay system has enabled a thorough analysis of the function of each discrete regulatory element of the Gatal gene. When a substitution mutation is introduced into the GATA motif in the G1HE core element in the context of G1BAC GFP reporter transgenic mice, the GFP expression was suppressed predominantly in the c-Kit-positive progenitor fraction, while GFP expression in Ter119-positive erythroblasts was maintained [37]. Similar reduction of endogenous Gatal expression in the progenitor fraction was observed in the $\triangle H S I \Delta N e o$ mice [37], in which the G1HE region was deleted from endogenous mouse Gatal locus [51]. These observations thus indicate that G1HE core exerts progenitor stage-specific enhancer activity, and that this activity largely depends on the GATA motif in the core region. These results further support the notion that G1BAC-based cis-regulatory element analysis is applicable to accurately dissect the physiological function of each cisregulatory element in the endogenous Gatal locus.

\section{Gatal gene suppression in HSCs}

The expression level of Gatal gene is suppressed at a low level in HSC, with abundant GATA2 expression detected in this fraction [35, 52]. It has been reported that forced GATA1 expression using retroviral transfection in HSC leads to the loss of self-renewal activity, suggesting that Gatal gene inactivation is crucial for maintenance of $\operatorname{HSC}[53,54]$.

Recently, we demonstrated that the $3.2-\mathrm{kb}$ genomic region intervening between G1HE core and dbGATA elements exerts DNA methylation-mediated silencing of Gatal gene in HSC fraction (G1MDR; Gatal methylationdetermining region; Fig. 6a) [35]. G1MDR sequences recruit DNA methyl transferase 1 (Dnmt1), which maintains high level of DNA methylation status throughout Gatal locus in HSC fraction (Fig. 6b left panel). Once HSC is differentiated into the erythroid progenitors, Dnmt 1 is released from Gatal locus, after which DNA demethylation proceeds. Demethylation of Gatal gene regulatory regions allowed abundant GATA2 binding to the GATAbinding site, inducing robust Gatal gene expression in the progenitor fraction (Fig. $6 \mathrm{~b}$, right panel). Targeted deletion of G1MDR sequences in the G1BAC-GFP transgenic context dramatically induces HSC-specific ectopic GFP expression [35]. This observation firmly supports our contention that G1MDR is important for Gatal gene suppression in the HSC fraction.

\section{Perspective}

In this review, we have described recent advances in the understanding of the spatiotemporal regulatory functions of each cis-acting element of Gatal and Gata2 genes, as well as the molecular basis of GATA factor switching during erythropoiesis. The Gatal BAC transgenic mouse system, coupled with homologous recombination-based mutation analysis, appears to be an important approach to the analysis and understanding of the functions of these discrete regulatory regions. ChIP-seq and ChIP-chip analyses have been providing valuable information for dissecting the function of GATA1 and GATA2 at various target loci during erythroid differentiation. However, many unresolved issues continue to confront our understanding of the hematopoietic GATA factors. Unraveling the molecular regulatory network directed by these hematopoietic GATA factors should provide further insights into normal 


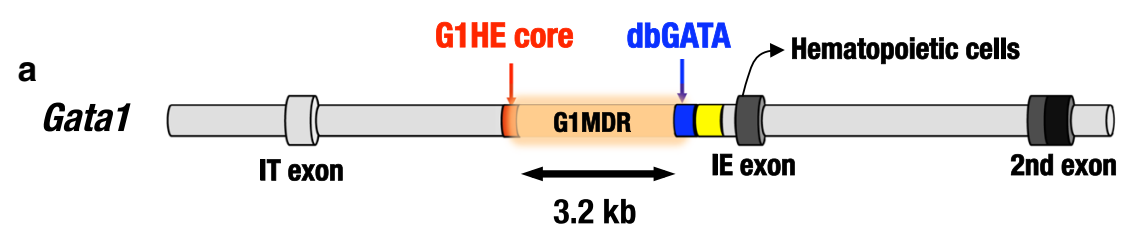

b
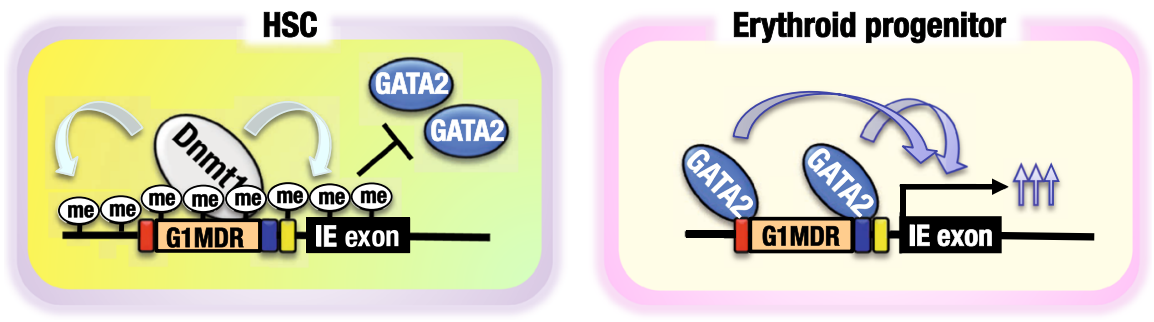

Fig. 6 a The 3.2-kb genomic region intervening between G1HE core and dbGATA elements (G1MDR; Gatal methylation-determining region) elicits Gatal gene silencing in HSCs. b G1MDR recruits DNA methyl transferase 1 (Dnmt1) and maintains DNA methylation

status of Gatal locus in HSC fraction (left panel). When HSCs are differentiated to the erythroid progenitors, Dnmt1 is released from Gatal locus, so that DNA demethylation proceeds, which allows GATA2 binding and results in Gatal gene activation (right panel)

hematopoiesis, as well as the pathological processes leading to GATA-related hematopoietic disorders.

Acknowledgments This work was supported through funding from JSPS KAKENHI Grant Number 24249015 (MY), 22118001(MY), 24590371 (TM), Takeda Foundation (to T.M. and M.Y.), Naito foundation (to M.Y.) and the Core Research for Evolutional Science and Technology from the JST (MY).

\section{References}

1. Yamamoto M, Ko LJ, Leonard MW, Beug H, Orkin SH, Engel JD. Activity and tissue-specific expression of the transcription factor NFE1 multigene family. Genes Dev. 1990;4:1650-62.

2. Orkin SH. GATA-binding transcription factors in hematopoietic cells. Blood. 1992;80:575-81.

3. Ohneda K, Yamamoto M. Roles of hematopoietic transcription factors GATA-1 and GATA-2 in the development of red blood cell lineage. Acta Haematol. 2002;108:237-45.

4. Ko LJ, Engel JD. DNA-binding specificities of the GATA transcription factor family. Mol Cell Biol. 1993;13:4011-22.

5. Patient RK, McGhee JD. The GATA family (vertebrates and invertebrates). Curr Opin Genet Dev. 2002;12:416-22.

6. Yamamoto M, Takahashi S, Onodera K, Muraosa Y, Engel JD. Upstream and downstream of erythroid transcription factor GATA-1. Genes Cells. 1997;2:107-15.

7. Ferreira R, Ohneda K, Yamamoto M, Philipsen S. GATA1 function, a paradigm for transcription factors in hematopoiesis. Mol Cell Biol. 2005;25:1215-27.

8. Fujiwara Y, Browne CP, Cunniff K, Goff SC, Orkin SH. Arrested development of embryonic red cell precursors in mouse embryos lacking transcription factor GATA-1. Proc Natl Acad Sci USA. 1996;93:12355-8.

9. Takahashi S, Onodera K, Motohashi H, Suwabe N, Hayashi N, Yanai $\mathrm{N}$, et al. Arrest in primitive erythroid cell development caused by promoter-specific disruption of the GATA-1 gene. J Biol Chem. 1997;272:12611-5.

10. Takahashi S, Shimizu R, Suwabe N, Kuroha T, Yoh K, Ohta J, et al. GATA factor transgenes under GATA-1 locus control

rescue germline GATA-1 mutant deficiencies. Blood 2000;96:910-6.

11. Tsai FY, Keller G, Kuo FC, Weiss M, Chen J, Rosenblatt M, et al. An early haematopoietic defect in mice lacking the transcription factor GATA-2. Nature. 1994;371:221-6.

12. Rodrigues NP, Janzen V, Forkert R, Dombkowski DM, Boyd AS, Orkin SH, et al. Haploinsufficiency of GATA-2 perturbs adult hematopoietic stem-cell homeostasis. Blood. 2005;106:477-84.

13. Ito E, Toki T, Ishihara H, Ohtani H, Gu L, Yokoyama M, Engel JD, Yamamoto M. Erythroid transcription factor GATA-1 is abundantly transcribed in mouse testis. Nature. 1993;362:466-8.

14. Onodera K, Takahashi S, Nishimura S, Ohta J, Motohashi H, Yomogida K, Hayashi N, Engel JD, Yamamoto M. GATA-1 transcription is controlled by distinct regulatory mechanisms during primitive and definitive erythropoiesis. Proc Natl Acad Sci USA. 199;94:4487-92.

15. Kobayashi E, Shimizu R, Kikuchi Y, Takahashi S, Yamamoto M. Loss of the Gatal gene IE exon leads to variant transcript expression and the production of a GATA1 protein lacking the N-terminal domain. J Biol Chem. 2010;285:773-83.

16. Yomogida K, Ohtani H, Harigae H, Ito E, Nishimune Y, Engel JD, et al. Developmental stage- and spermatogenic cycle-specific expression of transcription factor GATA-1 in mouse Sertoli cells. Development. 1994;120:1759-66.

17. Onodera K, Yomogida K, Suwabe N, Takahashi S, Muraosa Y, Hayashi $\mathrm{N}$, et al. Conserved structure, regulatory elements, and transcriptional regulation from the GATA-1 gene testis promoter. J Biochem. 1997;121:251-63.

18. Tsai SF, Strauss E, Orkin SH. Functional analysis and in vivo footprinting implicate the erythroid transcription factor GATA-1 as a positive regulator of its own promoter. Genes Dev. 1991;5:919-31.

19. Martin DI, Orkin SH. Transcriptional activation and DNA binding by the erythroid factor GF-1/NF-E1/Eryf 1. Genes Dev. 1990;4:1886-98.

20. Nishimura S, Takahashi S, Kuroha T, Suwabe N, Nagasawa T, Trainor C, Yamamoto M. A GATA box in the GATA-1 gene hematopoietic enhancer is a critical element in the network of GATA factors and sites that regulate this gene. Mol Cell Biol. 2000;20:713-23.

21. Motohashi H, Katsuoka F, Shavit JA, Engel JD, Yamamoto M. Positive or negative MARE-dependent transcriptional regulation 
is determined by the abundance of small Maf proteins. Cell. 2000;103:865-75.

22. Suzuki N, Ohneda O, Takahashi S, Higuchi M, Mukai HY, Nakahata T, Imagawa S, Yamamoto M. Erythroid-specific expression of the erythropoietin receptor rescued its null mutant mice from lethality. Blood. 2002;100:2279-88.

23. Tatsumi K, Yamamoto-Mukai H, Shimizu R, Waguri S, Sou YS, Sakamoto A, et al. The Ufm1-activating enzyme Uba5 is indispensable for erythroid differentiation in mice. Nat Commun. 2011;2:181.

24. Shimizu R, Ohneda K, Engel JD, Trainor CD, Yamamoto M. Transgenic rescue of GATA-1-deficient mice with GATA-1 lacking a FOG-1 association site phenocopies patients with X-linked thrombocytopenia. Blood. 2004;103:2560-7.

25. Shimizu R, Takahashi S, Ohneda K, Engel JD, Yamamoto M. In vivo requirements for GATA-1 functional domains during primitive and definitive erythropoiesis. EMBO J. 2001;20:5250-60.

26. Ohneda K, Shimizu R, Nishimura S, Muraosa Y, Takahashi S, Engel JD, et al. A minigene containing four discrete cis-elements recapitulates GATA-1 gene expression in vivo. Genes Cells. 2002;7:1243-54.

27. Shimizu R, Hasegawa A, Ottolenghi S, Ronchi A, Yamamoto M. Verification of the in vivo activity of three distinct cis-acting elements within the Gata1 gene promoter-proximal enhancer in mice. Genes Cells. 2013;18:1032-41.

28. Yien YY, Bieker JJ. EKLF/KLF1, a tissue-restricted integrator of transcriptional control, chromatin remodeling, and lineage determination. Mol Cell Biol. 2013;33:4-13.

29. Trainor CD, Omichinski JG, Vandergon TL, Gronenborn AM, Clore GM, Felsenfeld G. A palindromic regulatory site within vertebrate GATA-1 promoters requires both zinc fingers of the GATA-1 DNA-binding domain for high-affinity interaction. Mol Cell Biol. 1996;16:2238-47.

30. Trainor CD, Ghirlando R, Simpson MA. GATA zinc finger interactions modulate DNA binding and transactivation. J Biol Chem. 2000;275:28157-66.

31. Yu C, Cantor AB, Yang H, Browne C, Wells RA, Fujiwara Y, et al. Targeted deletion of a high-affinity GATA-binding site in the GATA-1 promoter leads to selective loss of the eosinophil lineage in vivo. J Exp Med. 2002;195:1387-95.

32. Lim LC, Swendeman SL, Sheffery M. Molecular cloning of the $\alpha$-globin transcription factor CP2. Mol Cell Biol. 1992;12: $828-35$.

33. Chae JH, Kim CG. CP2 binding to the promoter is essential for the enhanced transcription of globin genes in erythroid cells. Mol Cells. 2003;15:40-7.

34. Bosè F, Fugazza C, Casalgrandi M, Capelli A, Cunningham JM, Zhao Q, et al. Functional interaction of CP2 with GATA-1 in the regulation of erythroid promoters. Mol Cell Biol. 2006;26:3942-54.

35. Takai J, Moriguchi T, Suzuki M, Yu L, Ohneda K, Yamamoto M. The Gata $15^{\prime}$ region harbors distinct cis-regulatory modules that direct gene activation in erythroid cells and gene inactivation in HSCs. Blood. 2013;122:3450-60.

36. Suzuki N, Suwabe N, Ohneda O, Obara N, Imagawa S, Pan X, Motohashi $\mathrm{H}$, Yamamoto M. Identification and characterization of 2 types of erythroid progenitors that express GATA-1 at distinct levels. Blood. 2003;102:3575-83.

37. Suzuki M, Moriguchi T, Ohneda K, Yamamoto M. Differential contribution of the Gatal gene hematopoietic enhancer to erythroid differentiation. Mol Cell Biol. 2009;29:1163-75.

38. Whyatt D, Lindeboom F, Karis A, Ferreira R, Milot E, Hendriks $\mathrm{R}$, et al. An intrinsic but cell-nonautonomous defect in GATA-1overexpressing mouse erythroid cells. Nature. 2000;406:519-24.
39. Tsai FY, Orkin SH. Transcription factor GATA-2 is required for proliferation/survival of early hematopoietic cells and mast cell formation, but not for erythroid and myeloid terminal differentiation. Blood. 1997;89:3636-43.

40. Minegishi N, Ohta J, Suwabe N, Nakauchi H, Ishihara H, Hayashi $\mathrm{N}$, et al. Alternative promoters regulate transcription of the mouse GATA-2 gene. J Biol Chem. 1998;273:3625-34.

41. Minegishi N, Suzuki N, Yokomizo T, Pan X, Fujimoto T, Takahashi $\mathrm{S}$ et al. Expression and domain-specific function of GATA-2 during differentiation of the hematopoietic precursor cells in midgestation mouse embryos. Blood. 2003;102:896-5.

42. Khandekar M, Brandt W, Zhou Y, Dagenais S, Glover TW, Suzuki N, Shimizu R, Yamamoto M, Lim KC, Engel JD. A Gata2 intronic enhancer confers its pan-endothelia-specific regulation. Development. 2007;134:1703-12.

43. Johnson KD, Hsu AP, Ryu MJ, Wang J, Gao X, Boyer ME, et al. Cis-element mutated in GATA2-dependent immunodeficiency governs hematopoiesis and vascular integrity. J Clin Invest. 2012;122:3692-704.

44. Lim KC, Hosoya T, Brandt W, Ku CJ, Hosoya-Ohmura S, Camper SA, et al. Conditional Gata2 inactivation results in HSC loss and lymphatic mispatterning. $\mathrm{J}$ Clin Invest. 2012;122:3705-17.

45. Kaneko H, Shimizu R, Yamamoto M. GATA factor switching during erythroid differentiation. Curr Opin Hematol. 2010;17:163-8.

46. Suzuki M, Shimizu R, Yamamoto M. Transcriptional regulation by GATA1 and GATA2 during erythropoiesis. Int J Hematol. 2011;93:150-5.

47. Mukai HY, Suzuki M, Nagano M, Ohmori S, Otsuki A, Tsuchida $\mathrm{K}$, et al. Establishment of erythroleukemic GAK14 cells and characterization of GATA1 N-terminal domain. Genes Cells. 2013;18:886-98.

48. Suzuki M, Kobayashi-Osaki M, Tsutsumi S, Pan X, Ohmori S, Takai J, et al. GATA factor switching from GATA2 to GATA1 contributes to erythroid differentiation. Genes Cells. 2013;18:921-33.

49. Fujiwara T, O'Geen H, Keles S, Blahnik K, Linnemann AK, Kang YA, et al. Discovering hematopoietic mechanisms through genome-wide analysis of GATA factor chromatin occupancy. Mol Cell. 2009;36:667-81.

50. Moriguchi T, Suzuki M, Engel JD, Yamamoto M. GATA1 and GATA2 function in hematopoietic differentiation. In: Kondo M, editors. Hematopoietic stem cell biology. New York: Humana Press Inc; 2010. Pp 172-42.

51. McDevitt MA, Shivdasani RA, Fujiwara Y, Yang H, Orkin SH. A "knockdown" mutation created by cis-element gene targeting reveals the dependence of erythroid cell maturation on the level of transcription factor GATA-1. Proc Natl Acad Sci USA. 1997;94:6781-5.

52. Akashi K, Traver D, Miyamoto T, Weissman IL. A clonogenic common myeloid progenitor that gives rise to all myeloid lineages. Nature. 2000;404:193-7.

53. Iwasaki H, Mizuno S, Wells RA, Cantor AB, Watanabe S, Akashi K. GATA-1 converts lymphoid and myelomonocytic progenitors into the megakaryocyte/erythrocyte lineages. Immunity. 2003;19:451-2.

54. Togarrati PP, Suknuntha K. Generation of mature hematopoietic cells from human pluripotent stem cells. Int $\mathrm{J}$ Hematol. 2012;95:617-23. 